\title{
Jan Hecker-Stampehl Bloggen in der Geschichtswissenschaft als Form des Wissenstransfers
}

Die Publikationskultur in der Geschichtswissenschaft steckt in einem grundlegenden Veränderungsprozess. Bei der Erweiterung des publizistischen Repertoires von Historikerinnen und Historikern spielen Blogs eine große Rolle - unter der Überschrift einer „digital scholarship“ (hierzu Weller 2011, Kap. 4 "The Nature of Scholarship“) sind sie in vielen Disziplinen ein immer stärker genutztes Mittel zur Positionierung als Forscherin oder Forscher. In diesem Beitrag sollen die Chancen ausgelotet werden, die geschichtswissenschaftliche Blogs für die Vermittlung von Inhalten und Methoden des Fachs - fachintern und an die viel beschworene "breite Öffentlichkeit" - spielen können. Der Beitrag ist nicht empirisch angelegt, sondern versucht gewissermaßen, das Terrain "auszuloten“ und Potenziale aufzuzeigen. Grundlage sind medien- und kommunikationswissenschaftliche Auseinandersetzungen mit der Blogosphäre im Allgemeinen, aber auch fachspezifische Erörterungen zur Geschichtskultur und zur historischen Blogosphäre im Besonderen.

\section{Blogs und Digital Scholarship}

Es gibt nicht nur immer mehr Akademikerinnen und Akademiker, die Blogs lesen, es bloggen auch immer mehr von ihnen aktiv: "The target group or audience for these blogs is not necessarily only peers; the blogging activity can also be intended to disseminate research results to the public at large." (Kjellberg 2010) Wissenschaftliches Bloggen etabliert sich zusehends als weitere Form wissenschaftlichen Publizierens. In der anglo-amerikanischen Academia ist dies im Bereich der Geschichtswissenschaft schon so weit vorangeschritten, dass bloggende Historikerinnen und Historiker in einer populärwissenschaftlichen Zeitschrift sogar als Normalität beschrieben wurden (Poyntz 2010). Im deutschsprachigen Bereich ist dem nicht so - darum gibt es ja dieses Buchprojekt. Indes wächst die Zahl deutschsprachiger geschichts- und sozialwissenschaftlicher Blogs etwa auf der Plattform hypotheses.org in beeindruckendem Maße und in faszinierender fachlicher Vielfalt.

Für viele Akademikerinnen und Akademiker aus den Geistes- und Sozialwissenschaften scheint trotz dieser unbestreitbaren Dynamik die Feststellung 
zuzutreffen, dass sie in Hinblick auf die wissenschaftliche Blogosphäre passiv beobachtend verharren oder gar als indifferent erscheinen. Sie scheinen auf einen Appell zur Beteiligung zu warten - der aber nie erschallt (Quiroga 2011, S. 77). Das Heft des Handelns müssten nämlich sie in die Hand nehmen: Wer Teil der Blogosphäre werden will, muss selbst aktiv werden. In deutschsprachigen akademischen Milieus beinhaltet dies häufig noch, Kommentare zu erhalten wie „ist das ,richtige Wissenschaft'?" oder „das ist doch irrelevant und banal“. Blogs sind aber auch geradezu zum Inbegriff eines neuen digital geprägten Wissenschaftlerdaseins geworden - weil sie so einfach und schnell zu realisieren sind. Zugleich liegt in ihrer technischen wie inhaltlichen Unbeschränktheit auch ein Grund dafür, dass die Skepsis vielerorts noch anhält (Weller 2011, S. 5).

Wer „digital scholarship“ sagt, meint auf jeden Fall an zentraler Stelle - wenn auch nicht alleinig - Blogs. Der Wandel, der sich durch das Anwachsen der historyblogosphere abzeichnet, ist ein grundlegender und wird die Kommunikationskultur innerhalb des Fachs sowie vom Fach aus nach außen massiv verändern. Die anfängliche Zurückhaltung könnte auch ihr Gutes gehabt haben: Blogs sind durch mehrere Phasen von Skepsis und vehementer Kritik gegangen und vieles, was in der Frühphase über die Blogosphäre im Allgemeinen festgestellt wurde, hat sich abgeschliffen. Wenn es vor 15 Jahren überhaupt wissenschaftliche Blogs gab, haftete ihnen häufig der Geruch des Unseriösen an. Doch mittlerweile haben sich die verschiedensten Nutzungsformen von Blogs im akademischen Bereich etabliert. Eine davon ist ganz grundlegend die Kommunikation über fachliche Inhalte, das Schreiben über die eigene Forschungstätigkeit, das Informieren über fachliche Kontroversen und Debatten oder Hinweise auf Hintergrundwissen zu tagespolitischen Auseinandersetzungen (um nur einige Beispiele zu nennen). Mit dem wissenschaftlichen Bloggen steht der Geschichtswissenschaft ein neuer Kanal zur Verfügung, der die Chance in sich birgt, neue Verbindungen zwischen der fachlichen und der breiten Öffentlichkeit zu knüpfen - oder alte zu erneuern.

\section{Wissenstransfer innerhalb der Geschichtswissenschaft}

Die Geschichtswissenschaft ist anno 2013 in viele Unterdisziplinen ausdifferenziert. Dieser Prozess setzte im Prinzip mit der Verwissenschaftlichung der Geschichtsschreibung ein, wenn auch die meisten Verzweigungen des Fachs in der Zeit nach dem Zweiten Weltkrieg entstanden sind. Gelegentlich wird die im Laufe der Zeit entstandene Pluralität von Diskursen noch zusammengeführt, etwa beim Deutschen Historikertag. In der Alltagspraxis pflegen jedoch 
Mediävistinnen und Mediävisten eine eigene Diskussion, die Osteuropäische Geschichte gilt nahezu als eigenes Fach, die Lateinamerikahistorikerin wird nur selten Berührungspunkte mit den Gegenständen des Nordeuropahistorikers finden. Die hochgradige Spezialisierung, die in den Qualifikationsarbeiten abverlangt wird, führt dazu, dass Expertenwissen häufig in kleinteiligen Milieus akkumuliert und diskutiert wird. Hier entstehen methodisch wie von der Quellenerschließung her oft sehr ambitionierte Arbeiten, die aber ein eher kleines Publikum erreichen. Auf der anderen Seite stehen die immer stärkere Beschäftigung mit der deutschen Geschichte oder gleich die Verlagerung auf Phänomene der Globalgeschichte, nicht zuletzt auch postkoloniale Ansätze. Diese knappen Hinweise deuten schon an, dass es widersprüchliche Trends gibt, dass Forderungen erhoben werden - wie etwa jüngst nach einer stärker transnational ausgerichteten Geschichtswissenschaft - die in Teilen des Fachs längst gängige Praxis sind. Man weiß schlichtweg zu wenig übereinander. Allein fachintern sollten Blogs eine viel größere Rolle im Wissenstransfer spielen und im Abbau von Hierarchien hilfreich sein.

Die Aufsplitterung des Fachs in einzelne Epochen, gar Subdisziplinen wird auch in der historyblogosphere nicht aufgehoben - dies soll und kann sie auch gar nicht. Allerdings sollte man der Illusion einer - so nie dagewesenen - Totalität des Faches, einer allumfassenden internen disziplinären Öffentlichkeit, gar nicht erst erliegen. Dennoch: Eher als die teilweise stark spezialisierten wissenschaftlichen Zeitschriften, die sich auch an eigene Teilöffentlichkeiten richten, bieten Blogs die Chance, Wissen aus den verschiedenen Teilen des Fachs in eine größere Fachöffentlichkeit zu kommunizieren. Die Knotenpunkte wären beispielsweise als Themenpools oder in Form von "Methoden-Sharing“ vorstellbar. Einen großen undifferenzierten Einheitsbrei will niemand. Doch zu wenig verschaffen wir uns innerhalb des Fachs Klarheit über das, was über diese Aufsplitterungen hinweg verbindet. Gerade Gruppen-Blogs mit größerer Autorenschaft könnten Entwicklungen im Fach in ihren spezifischen Ausformungen in verschiedenen Teilen der Disziplin aufgreifen, um methodisch-theoretische Diskussionen auf andere Weise zu führen und zu bündeln als bisher. Weitere Vorteile bieten in diesem Zusammenhang die Kategorisierung und Verschlagwortung der Beiträge durch Tagging. Die Verknüpfung der einzelnen Knotenpunkte zu einem großen Wissensnetz kann durch das Verlinken und die Trackback-/Pingback-Funktion vorangetrieben werden. Insofern könnten Blogs dabei helfen, so manche Forscherin und manchen Forscher aus dem fachlichen Inseldasein herauszulösen.

Ein nicht zu unterschätzender Aspekt bei der Frage danach, welches Wissen hier vermittelt wird, ist mit dem vermeintlich problematischen subjektiven Charakter von Blogs verknüpft. Das Subjektive (siehe Beitrag von J. Schreiner) und die Geschichtswissenschaft, die so sehr mit der komplexen Problematik 
"historische Objektivität" ringt - das scheint nicht recht zusammenzupassen. Dabei wird hier nur transparenter gehandhabt, was doch ohnehin spätestens seit der Konstruktivismus-Debatte und den Debatten um die Geschichtswissenschaft in der Postmoderne klar geworden ist: Wir als Historikerinnen und Historiker haben hochgradig subjektiv geprägte Zugänge zu unseren Themen. Wir konstruieren unsere Themen und unsere Quellenkorpora und finden sie nicht fertig vor. Blogs legen diesen Umstand noch deutlicher frei und können so ein Bild davon zeichnen, wie geschichtswissenschaftliche Forschungspraxis funktioniert. Weller spricht im Zusammenhang von wissenschaftlichen Blogs auch von dem Phänomen des ,thought share“, um diese Subjektivität herauszustreichen (Weller 2011, S. 67).

\section{Wissenstransfer in die Öffentlichkeit}

Zugleich gelangen nicht allzu viele Themen aus der fachlichen Diskussion in die Öffentlichkeit. Die traditionellen Medien kanalisieren das Interesse auf eine bestimmte Auswahl von Themen, überspitzt gesagt, primär auf das Dritte Reich, den Zweiten Weltkrieg, die deutsch-deutsche Teilungsgeschichte - und ein wenig Drumherum. Die klassischen Medien fallen durch eine inhaltliche Engführung auf und richten sich zudem primär an ein nicht-fachliches Publikum. Das Interessante an der historyblogosphere ist dagegen ihre Hybridität. Geschichtswissenschaftliche Blogs verfügen über ein enormes Potenzial für den Wissenstransfer - nicht nur in andere Teilöffentlichkeiten des Fachs, sondern auch in die allgemeine Öffentlichkeit hinein. Das Interesse an geschichtlichen Themen ist nach wie vor ungebrochen, wenn es nicht gar noch weiter wächst. An der Aufmerksamkeit, die historische Themen in Fernsehen, Radio und Kino finden, kann man schon länger nicht mehr vorbei sehen; auf die immense Nachfrage nach historischen Themen und die Beteiligung zahlreicher „Amateurinnen und Amateure", ob auf Wikipedia, oder mit eigenen Geschichtsseiten oder auch Blogs im Netz (auch Amateurinnen und Amateure sind Teil der historyblogosphere!) ist bereits hingewiesen worden (stellvertretend: Cohen/Rosenzweig 2006; Rosenzweig 2011). Doch im Unterschied zur Open-Access-Bewegung, welche für die Öffnung der Wissenschaft ebenfalls relevant ist, passiert beim wissenschaftlichen Bloggen noch etwas Anderes: Durch Open Access ändert sich die Zugänglichkeit wissenschaftlicher Inhalte; Barrieren wie kostenpflichtige Abonnements, Verfügbarkeit von Nutzungslizenzen in Universitäts- und Bibliotheksnetzwerken und Passwortschutz entfallen. Das Genre des wissenschaftlichen Textes mit Fußnotenapparat etc. bleibt jedoch in der Regel gleich. 
Bloggen hingegen ist eine distinkt andere Form der Autorschaft, welche verschiedene Medien und Textgenres aggregiert und welche nicht-linear und konzentrisch (Stichworte: Hyperlinks, Blogroll, Pingbacks, Embedding) funktioniert (Weller 2011, S. 67). Bloggen ergänzt traditionelle wissenschaftliche Publikationsformate und wird letztere auch verändern - aber nicht vertreiben. Beim Bloggen kommt im Rahmen des Wissenstransfers der in der Philosophie des Web 2.0 so prominente Gedanke des „Sharing“ zum Zuge. Dabei kommen Blogs den Lese- und Konsumgewohnheiten der breiteren Öffentlichkeit wesentlich stärker entgegen als wissenschaftliche Papers. Das über Blogs betriebene „knowledge sharing“ geht - ganz im Sinne der „non-profit“/ open-source-Mentalität - nicht von einem sich unmittelbar auszahlenden Nutzen aus. Wissenschaftsbloggerinnen und -blogger können sich kaum Profit im materiellen Sinne erhoffen, wenngleich sie nicht aus purem Altruismus agieren. „Knowledge sharing“ über Blogs ist indes momentan noch kein voll akzeptierter Baustein zum Aufbau sozialen Kapitals in der Wissenschaftslandschaft. Meine Prognose ist, dass ein eigener Fachblog oder die Beteiligung an einem wissenschaftlichen Gruppenblog über kurz oder lang für viele (nicht alle) zum Portfolio dazugehören wird (mehr zu Aspekten des Reputationsgewinns und der Bereitschaft zum "knowledge sharing" in Online-Communities: Shu/Chuang 2011). Der Skepsis, die Andreas Fahrmeir diesbezüglich äußert (vgl. seinen Beitrag in diesem Band), sollte man dennoch Gehör schenken. Blogs werden die klassische Hierarchie der akademischen Publikationskanäle schwerlich umstoßen, sie sind eher ein Addendum und ein Mittel, um das, was in den umfänglicheren und fachjargonbeladeneren Textgenres für die fachliche Community aufbereitet wird, in andere Foren zu tragen. Gleichwohl: Es werden und müssen gar nicht alle bloggen, doch ist vorstellbar, dass Forscherinnen und Forscher immer wieder punktuell und an verschiedenen Orten an Blogs beteiligt sind. Ein zeitlich begrenztes Forschungsprojekt informiert womöglich in Blogform über seine Arbeit, es werden in Zukunft wahrscheinlich mehr Gruppenblogs zu bestimmten Subdisziplinen oder Spezialthemen aufkommen, für die hie und da Beiträge beizusteuern sind, Konferenzblogs sind ein weiteres Beispiel.

Es gibt Stimmen, welche eine Verlagerung der fachlichen Diskussionen in die sozialen Netzwerke vermuten, doch dürften diese eher eine Brückenfunktion zu Blog-Inhalten wahrnehmen. Die Kürze eines Tweets oder eines Postings in Facebook, Google+ etc. reicht für eine ausführlichere Auseinandersetzung mit Inhalten kaum aus. Die unter internetaffinen Wissenschaftlern zunehmend beliebte Plattform Twitter dient als Verweismedium jedoch dazu, auf Blogbeiträge aufmerksam zu machen und Diskussionen anzustoßen. Trotz der Kürze sind indes selbst auf diesen Medien pingpongartige Debatten möglich, doch kommen die Tweets und Postings in solchen Zusammenhängen selten ohne 
Links auf weiterführende Lektüre aus. Die URL-Shortener, mit deren Hilfe die Links eingebaut werden, benötigen dabei immer wieder neues Futter - etwa in Form von Verweisen auf Blog-Beiträge. Diese Chance zu neuer und möglicherweise stärker assoziativer Referentialität von Blogs könnte man auch in Hinblick auf andere Online-Inhalte weiterdenken. Die oft kritisierten kurzen Aufmerksamkeitsspannen in der digitalen Lese- und Klick-Kultur sind das eine, das andere ist die Möglichkeit, auf ganz ungeahnten und ungewohnten Wegen zu neuen Inhalten im Netz geführt zu werden. Ein Blog-Beitrag, der eine digitale Quellensammlung vorstellt und kritisch kommentiert, wird auf diese selbst, aber auch auf andere Beispiele und verwandtes Material verlinken. Eine kritische Einlassung über einen Wikipedia-Eintrag wird auf diesen verweisen, aber hierin liegt zumindest das Potenzial, welches Blogs durch ihren oft reflektierenden Charakter aufweisen - es wird oder sollte nicht bei einem bloßen Verweis bleiben. Wie wenige andere Foren ermöglichen Blogs neben der Verlinkung auch eine Kommentierung dessen, worauf man verweist. Es geht dabei weniger um eine technische Möglichkeit (dies ginge in Online-Diskussionsforen ja auch!), sondern darum, dass Blogs durch das Fehlen einer Registrierungspflicht für Leserinnen und Leser (wie etwa bei den meisten Foren) offener zugänglich sind. Zudem können wissenschaftliche Blogs durch die Impressumspflicht und durch das Interesse ihrer Betreiberinnen und Betreiber, ihren Namen mit dem Produkt zu verbinden, ein gewisses soziales Kapital aufweisen, dass der Schar an anonym oder unter einer Vielzahl bizarrer Pseudonyme agierenden ForenUser fehlt. Damit stehen wissenschaftliche Blogs auch für Zuverlässigkeit und Nachvollziehbarkeit, sowie gegebenenfalls namentlich zu adressierenden Widerspruch. Die Erfahrungen zeigen, dass Wissenschaftsblogs somit einer der größten Unsicherheiten, die etwa Studierende in der Begegnung mit dem Internet berichten, begegnen könnten. Sie halten eine Antwort auf die Frage bereit: "Wo finde ich im Internet eigentlich seriöse und verlässliche Informationen?" Wissenschaftsblogger sollten daran arbeiten und sich geradezu dazu berufen fühlen, die Bereitstellung substanzieller und vertrauenswürdiger wissenschaftlicher Erkenntnisse im Netz zu ermöglichen.

\section{Charakteristika von Blogs ...}

Blogs sind überall und kaum zu übersehen, auch wenn sich die deutschsprachige Geschichtswissenschaft noch schwer mit ihnen tut. Dabei spricht viel für sie: „Blogs can also be used as tools to facilitate research, collaboration, and the sharing of knowledge. These media offer an avenue for faculty members to engage a society in which use of the Internet has become common and routine." 
(Powell et al. 2012, S. 272) Blogs sind also längst „in der Mitte der Gesellschaft angekommen" - was die Schranken für Leserinnen und Leser senken könnte, die die „klassischen Formate“ als schwierig oder gar abschreckend empfinden. Hierzu trägt jedenfalls im deutschen Sprachraum der noch zu weit verbreitete hermetische und komplexe Fachjargon bei. Verständlich, griffig und möglicherweise so knapp wie in einem Blog-Beitrag zu schreiben, ist noch nicht allerorten ,gängige Währung' in der akademischen Meritokratie. Verdienste erwirbt man sich in der Regel immer noch mit einer umfangreichen Monografie. Dieses Format wird auch gar nicht verschwinden - oder gar weichen müssen. Für das Ausbreiten, Durchdringen und Analysieren großer Quellenbestände wird man weiterhin ein gewisses Maß an Platz benötigen. Von daher werden Blogs nicht Ersatz, sondern ein zusätzlicher Kommunikations- und Publikationskanal werden - und das vermutlich in immer weiter ansteigendem Maße (Walker 2007, S. 136).

Übliche Erörterungen zu Blogs verweisen auf die Spontaneität, die Aktualität, den Tagebuch-Charakter, Subjektivität, kurze Texte, Blog-Manifestos fordern dazu auf, das Scheitern beim Bloggen mit einzuplanen. Schnelle Interaktion ist gefragt, viele Analysen heben auch auf den quasi-journalistischen Charakter von Blogs ab (Schmidt 2006, S. 199 ff.; Ebersbach et al. 2011, S. 61 ff.; Porombka 2011). Manche Autorinnen und Autoren meinen gar, mit zeithistorischen Blogs ließe sich unmittelbar in aktuelle Debatten eingreifen, und sehen die Vermittlungsarbeit primär auf Foren des politischen Diskurses und internationaler Konflikte (Cole 2011, S. 666 ff.). Nach einer Einteilung in einem Überblickswerk zum Social Web würde die Facette des wissenschaftlichen Bloggens, die hier umrissen wird, etwas profan wie folgt kategorisiert: "Infoblogs informieren themenspezifisch“. Zudem sind viele geschichtswissenschaftliche Blogs Textblogs - die Disziplin ist nun einmal sowohl von ihrem Quellenmaterial wie von ihrer akademischen Praxis her sehr textlastig (Kategorien nach Ebersbach et al. 2011, S. 65). Doch liegt für Geschichtsblogs der Wandel nicht allein in der Schreibform - in der Tat greift eine Sicht auf das Bloggen als „Schreibwerkstatt“ oder als „anderer Schreibmodus“ zu kurz. Allein die multimedialen Elemente, die in einem Blog-Post verknüpft werden können - und denen immer noch die Kritik der skeptisch gebliebenen nicht-bloggenden Kolleginnen und Kollegen gilt - erlauben eine ganz neue Art der Vermittlung historischer fachlicher Inhalte (Quiroga 2011, S. 68, 77). Dabei kommen Blog-Posts, welche verschiedene mediale Inhalte verbinden, den Konsumgewohnheiten vieler Rezipientinnen und Rezipienten näher. Sie können etwas erfüllen, was in keiner Monografie und in keinem traditionellen Aufsatz möglich wäre, und auch nicht in den monodirektionalen klassischen Medien: nämlich, Geschichte mit allen Sinnen zu vermitteln und zugleich Interaktion zu ermöglichen. Es muss aber eingeräumt werden, dass hier noch 
einiges zu leisten ist und eine solche Idealform des Blog-Beitrags noch nicht die Normalität in der historyblogosphere darstellt.

\section{... und welche davon für den Wissenstransfer genutzt werden könnten}

Neben den multimedialen Möglichkeiten ist es aber auch die Aufhebung der Grenzen zwischen interner und externer Fachkommunikation, welche den Blogs ihre Stärke als Medium des Wissenstransfers eröffnet. In der Blogosphäre ist die Grenze zwischen verschiedenen Leserschaften nicht erkennbar, nicht steuerbar und eine Grenzziehung auch gar nicht mehr erwünscht. Pierre Mounier schreibt in diesem Zusammenhang davon, wie Historikerinnen und Historiker auf dem eigenen Blog in einem "atelier ouvert" arbeiten, wo man ,zusehen' kann, wie Forschung passiert (vgl. auch den Beitrag von Mounier in diesem Band). Viele Forschungsblogs, welche begleitend zu einer Qualifikationsarbeit oder einem größeren Projekt entstehen, nehmen genau diese Rolle ein: Sie machen Vorgehensweisen und methodische Stolpersteine öffentlich, sie erörtern arbeitstechnische Herausforderungen, etwa die richtige Technik zum Abfotografieren von Archivquellen mit Digitalkameras (Zimmermann 2012). Der vermittelnde Charakter besteht also nicht nur darin, dass Gegenstände der historischen Forschung ausgebreitet werden, sondern in einem viel höheren Maße als früher die Tür zur,Werkstatt des Historikers' geöffnet und Einblick gewährt wird. Kolleginnen und Kollegen, die an ähnlichen Problemen sitzen, könnten durch Blogs viel früher auf parallel laufende Projekte ähnlicher Art stoßen und sich mit diesen vernetzen. Gerade, wo die Forschungsblogs offen und selbstkritisch gefasst sind, zeigen sie auf, wo und wie Stolpersteine das Arbeiten an dem eigenen Thema beeinflussen, aufhalten, schwierig machen. Solche Forschungsblogs können das Prozesshafte offenlegen und damit methodische Diskussionen stimulieren, aber auch Beispiele und Anregungen für andere Generationen von Forscherinnen und Forschern geben. Geschichte schreibt sich eben nicht von selbst, und geschichtswissenschaftliche Forschungsarbeiten ebenso wenig. Hier wäre also ein Wissenstransfer im Sinne einer Open Science erkennbar.

Diesem Verständnis folgend könnten Barrieren zwischen der innerfachlichen und der nach außen gerichteten Kommunikation abgebaut werden: „Finalement, il évoque un aspect de la recherche,en train de se faire $[. .$.$] , qui$ intéresse aussi bien ses collègues les plus immédiats [...] et en même temps un plus large public intéressé pour une raison ou une autre par le thème traité." Indem sie es wagen, ihre wissenschaftliche Arbeit im Stadium der Unfertigkeit 
zur Diskussion zu stellen, können bloggende Historikerinnen und Historiker Studierenden und jüngeren Forscherinnen und Forschern Einblick in den Arbeitsprozess geben und sie zur eigenen Arbeit motivieren. Die Freiheit, sich nur einzelne methodische oder inhaltliche Aspekte herauszugreifen und hinsichtlich der Themenwahl, des Veröffentlichungsrhythmus', der Länge des Beitrags und des jeweils gewählten Stils nicht festgelegt zu sein, erlaubt ihnen, eine hybride Leserschaft anzuziehen, die auf nur einem einzigen Kanal gar nicht erreicht werden könnte (Mounier 2011, S. 102 f., Zitat: S. 102).

\section{Geschichte in der Öffentlichkeit - Chancen für geschichtswissenschaftliche Blogs}

Geschichtliche Themen werden nicht nur, wie bereits erwähnt, in der Öffentlichkeit zunehmend stärker nachgefragt, es vollzieht sich auch ein Wandel darin, wie diese Themen abgerufen oder rezipiert werden. Geschichte wird zunehmend zum Konsumobjekt, einem der erfolgreichsten in der kommerzialisierten Vermarktung von Bildungsinhalten obendrein. An dieser Stelle sind Historikerinnen und Historiker in besonderem Maße gefragt. Wir können uns der Tatsache nicht verschließen, dass Geschichte eben immer stärker konsumiert wird und es Nachfrage nach der ,Ware Geschichte' gibt. Geschichte ist ,unterhaltsam ' geworden und die leichte Zugänglichkeit zu entsprechenden Beiträgen jedweder Art und Qualität im Netz muss kritisch betrachtet werden (vgl. hierzu die Beiträge in Hardtwig/Schug 2009). In der Tat: Historikerinnen und Historiker sollten die Trivialisierung und Engführung von Geschichte, egal, wo sie stattfindet, kritisieren. Sich aber nach Abfeuern der Kritik wieder gemütlich in den Ohrensessel der Tradition zurückzulehnen und nichts weiter zu unternehmen, nur um bei nächster Gelegenheit erneut loszuzetern: Damit wird man diesen Herausforderungen nicht gerecht. In einer multipolaren Kommunikationslandschaft und Medienwelt bieten Blogs eine nicht zu unterschätzende Chance, um die Geschichtswissenschaft wieder in die öffentliche Vermittlung von Geschichte einzubringen. „Academics have created valuable techniques aimed at preserving the integrity and, as far as is possible, the objectivity of their writing. But in this new world, where barriers between the ivory tower and the public are being eroded by the internet, and where, it turns out, the public is sometimes deeply interested in the results of our researches, historians have to ask themselves if they want always to leave the popularization of their findings to journalists, generalists and (sometimes) mere rhetoricians." (Cole 2011, S. 670)

Geschichtswissenschaftliche Blogs sollten also nicht zuletzt als an die Gesellschaft gerichtete wissenschaftliche Dienstleistung verstanden werden: Sie 
informieren aus ihrem jeweiligen Fachgebiet, punktuell, aktuell und mit einem Blick über die fachliche Sphäre oder spezielle Subdisziplin hinaus. Zudem helfen sie, sich in der unübersichtlichen Welt der digitalen, online stattfindenden Geschichtswissenschaft zu orientieren. Die bloggende Historikerin empfiehlt best-practice-Beispiele, weist auf neue Angebote im Netz hin, teilt eigene Erfahrungen, gibt Benutzungstipps etc. Der bloggende Historiker weist auf Geschichtsdebatten hin, die in anderen Ländern und Regionen stattfinden, die sich nicht allen Usern sprachlich erschließen. Hier könnten in noch viel stärkerem Maße fachliche wie sprachliche ,Übersetzungsleistungen' erhofft werden. Es bleibt dabei abzuwarten, ob geschichtswissenschaftliche Blogs in allen Fällen die klassischen Vorstellungen, die über Blogs als hochgradig diskursive Foren existieren, erfüllen werden. Die Philosophie des Web 2.0 würde das Toben intensiver Kommentarschlachten erwarten lassen, den kontroversen Austausch von Thesen - gleichwohl, es ist (noch?) nicht überall so. Lese- und Diskussionsgewohnheiten müssen sich in den Geisteswissenschaften in Bezug auf Blogs im Laufe der nächsten Jahre erst noch ändern (vgl. Beitrag und Kommentare bei Baillot 2012) - und selbst dann dürfte es bei dem Bild vom „Blogger als Monarch“ und der „Aristokratie der Kommentatoren“ vom Grundsatz her bleiben (Friedrich 2012, S. 92). Die Fachdiskussion wird sich nicht in die Blog-Kommentarfelder verlagern - allenfalls zu einem geringen Teil. Das tut aber auch überhaupt nicht Not - schließlich werden wir weiterhin auf Konferenzen fahren und die Kolleginnen und Kollegen dort treffen. Warten Sie schon mal gespannt auf die ersten Gespräche in den Kaffeepausen, in denen es dann heißt „ich habe dazu letztens ihren sehr interessanten Blog-Beitrag gelesen - was ich dazu anmerken wollte: ..."!

Als Instrumente des Wissenstransfers und mit Hinblick auf ihre Rolle im Rahmen der Public History könnten Blogs aber durchaus die Funktion von Materialpools übernehmen, die durch die Verschlagwortung mithilfe der Tags, aber auch durch die Sortierung in Kategorien durchsuchbar sind. Eine enzyklopädische Abdeckung aller möglichen Bereiche ist hier sicher nicht machbar oder überhaupt wünschenswert - der Wikipedia soll und wird die historyblogosphere keine Konkurrenz machen. Das Genre des Blogs gibt eine solche Ausrichtung auch gar nicht her, bietet dafür aber andere Möglichkeiten. Blogs als sich stets aktualisierende Angebote bieten sich z. B. durch Duktus, Zugänglichkeit und die konzise Knappheit der einzelnen Beiträge dazu an, historische Inhalte mit aktuellen Anlässen zu verknüpfen. Ohne einem atemlosen Aktualismus das Wort zu reden: Blogs könnten so etwas wie den gesellschaftlichen Nutzen von Geschichtswissenschaft wieder evident machen, indem sie Expertise und Hintergrundwissen in die öffentliche Debatte einbringen, für die dem Journalismus meist die Zeit oder die Perspektive fehlen. 


\section{Fazit}

Die geschichtswissenschaftliche Blogosphäre birgt also die Chance des Brückenbaus zwischen akademischer Welt und öffentlichem Geschichtsinteresse in sich. Historikerinnen und Historiker sollten - in welcher Form auch immer es ihnen liegt - Blogs als einen selbstverständlichen Zweig ihrer Arbeit begreifen und die Chance erkennen, die sich für den Wissenstransfer bietet. Die Geschichtswissenschaft könnte mithilfe der historyblogosphere in ihrer Vielfalt wieder sichtbarer werden und das politische und wirtschaftliche Geschehen mit historischem Hintergrundwissen unterfüttern. Die Relevanz geschichtswissenschaftlicher Forschung könnte somit deutlich gemacht werden, und die Mauer zwischen „Elfenbeinturm“ und öffentlicher Diskussion wenigstens wieder ein Stück weit abgebaut werden. Blogs bergen die Chance, das Fach in einer sehr viel größeren Bandbreite nach außen zu repräsentieren. Gewiss: Historikerinnen und Historiker sind in den klassischen Medien lange schon präsent gewesen, doch sind es überwiegend über die Fächergrenzen hinweg bekannte (in der Tat häufig: männliche!) Vertreter, denen diese Form der Selbstdarstellung und Wissensvermittlung zugänglich ist. „Virtually nothing most historians could have written 40 years ago could have hoped to have a million readers over a single month, with some rare exceptions [...]. Few historians could have hoped to crack the editorial pages of prestigious newspapers, save if they were at elite institutions and already had the ear of the powerful. The digital revolution is undermining many publication practices that have been taken for granted for two centuries" (Cole 2011, S. 669).

Mit dem Ausbau der historyblogosphere wächst das Potenzial, um die Geschichtswissenschaft in verschiedenen Teilen der öffentlichen Sphäre stärker zu verankern. Bloggende Historikerinnen und Historiker müssen nicht gleich ein Millionenpublikum erreichen, um das von ihnen erarbeitete Wissen an eine größere und hybride Leserschaft zu vermitteln. Doch sollten sie in einem höheren Maße die Chance für einen neuen kommunikativen Pluralismus wahrnehmen und über Blogs ihre Forschung, ihre Standpunkte und ihre Freude an der Geschichte und der Geschichtswissenschaft unmittelbar an diese hybride Leserschaft vermitteln. In diesem Sinne: Raus aus dem Ohrensessel - hinein ins Web 2.0 ! 


\section{Literatur}

Anne Baillot, Meta-Blogging, Blog-Beitrag 20.9.2012, in Blog: Digital Intellectuals. A blog accompanying the production of the digital edition „Letters and texts. The intellectual Berlin around 1800“ [digitalintellectuals.hypotheses.org/ 750], eingesehen 21.9.2012.

Daniel Cohen/Roy Rosenzweig: Digital History. A Guide to Gathering, Preserving, and Presenting the Past on the Web. Philadelphia, PA 2005.

Juan Cole: Blogging Current Affairs History, in: JContH 46 (2011:3), S. 658-670.

Anja Ebersbach/Markus Glaser/Richard Heigl: Social Web. 2. Aufl. Stuttgart 2011.

Jörg Friedrich: Kritik der vernetzten Vernunft. Philosophie für Netzbewohner. Hannover 2012.

Wolfgang Hardtwig/Alexander Schug (Hrsg.): History Sells! Angewandte Geschichte als Wissenschaft und Markt. Stuttgart 2009.

Sara Kjellberg: I Am a Blogging Researcher: Motivations for Blogging in a Scholarly Context, in: First Monday 15 (2010:8) [firstmonday.org/htbin/cgiwrap/bin/ ojs/index.php/fm/article/view/2962/2580], eingesehen 21.8.2012.

Pierre Mounier: Ouvrir l'atelier de l'historien. Médias sociaux et carnets de recherche en ligne, in: Revue d'histoire moderne et contemporaine 58 (2011:5), S. 101-110.

Stephan Porombka: Schreiben unter Strom. Experimentieren mit Twitter, Blogs, Facebook \& Co. Mannheim 2011.

Douglas A. Powell/Casey J. Jacob/Benjamin J. Chapman: Using Blogs and New Media in Academic Practice, in: Innovative Higher Education 37 (2012:4), S. 271-282.

Nick Poyntz: History Blogs, in: History Today 60 (2010:5), S. 37.

Nicolás Quiroga: Blogs de historia: usos y posibilidades, in: Historia Crítica 43 (2011), S. 62-80.

Roy Rosenzweig: Clio Wired: The Future of the Past in the Digital Age. New York 2011.

Jan Schmidt: Weblogs. Eine kommunikationssoziologische Studie. Konstanz 2006.

Wesley Shu/Yu-Hao Chuang: Why People Share Knowledge in Virtual Communities, in: Social Behavior and Personality. An International Journal 39 (2011:5), S. 671-690. 
Jill Walker: Blogging From Inside the Ivory Tower, in: Axel Bruns/Joanne Jacobs (Hrsg.): Uses of Blogs. New York 2007, S. 127-138.

Martin Weller: The Digital Scholar. How Technology Is Transforming Scholarly Practice. London/New York 2011.

Robert Zimmermann, Fotografieren im Archiv - Teil 1, Blogbeitrag 9.5.2012, in: Blog: Das umstrittene Gedächtnis. Erinnerungspraktiken in Skandinavien, [umstrittenesgedaechtnis.hypotheses.org/77], eingesehen 23.9.2012. 
